\title{
Correction to: The High-Order Mixed Mimetic Finite Difference Method for Time-Dependent Diffusion Problems
}

\author{
Gianmarco Manzini ${ }^{1} \cdot$ Gianluca Maguolo ${ }^{2} \cdot$ Mario Putti $^{3}$ \\ Published online: 16 August 2019 \\ (c) Springer Science+Business Media, LLC, part of Springer Nature 2019
}

\section{Correction to: Journal of Scientific Computing https://doi.org/10.1007/s10915-019-01002-4}

The original version of this article unfortunately contained the following errors:

In Section 2, the reference to equations should be (see $2 a-2 e$ ) instead of (see $2 a-2 d$ ).

In equation (34a), the symbol $\phi_{h}$ should be $\phi^{\mathrm{I}}$.

In the proof of Theorem 1, the reference to equation should be (32) instead of (34). Also, the penultimate sentence in the theorem proof should read as "We then note that $\widehat{\mathbf{u}}_{h}$ and $\widehat{p}_{h}$ are continuously differentiable with respect to $t$ and solve the problem obtained by taking the derivative of (33)."

The superfluous (t) in equation (41) should be removed (i.e., $\left[((\operatorname{div} \mathbf{u})(t))^{\mathrm{I}}(t), q_{h}\right]_{Q_{h}}$ should be changed as $\left.\left[((\operatorname{div} \mathbf{u})(t))^{\mathrm{I}}, q_{h}\right]_{Q_{h}}\right)$.

The expression $\frac{\partial p}{\partial t}$ in theorems 2-4 should be changed to $\frac{d p}{d t}$.

The double vertical bars || || in few equations should be changed to triple vertical bars ||| |||.

In inequality (46), the first two norms should have subscript $X_{h}$ instead of $Q_{h}$.

Finally, in the reference section, the author name should be 'Beirão da Veiga, L.' instead of 'da Veiga, Beirão L.'.

The original article has been corrected.

Publisher's Note Springer Nature remains neutral with regard to jurisdictional claims in published maps and institutional affiliations.

The original article can be found online at https://doi.org/10.1007/s10915-019-01002-4.

Mario Putti

putti@math.unipd.it

Gianmarco Manzini

gmanzini@lanl.gov

Gianluca Maguolo

gianlucamaguolo93@gmail.com

1 Group T-5, Theoretical Division, Los Alamos National Laboratory, Los Alamos, NM, USA

2 Department of Information Engineering, University of Padua, via Gradenigo 6, Padua, Italy

3 Department of Mathematics "Tullio Levi-Civita", University of Padua, via Trieste 63, Padua, Italy 